\title{
LOCAL PROJECTIVE MODEL STRUCTURES ON SIMPLICIAL PRESHEAVES
}

\author{
BENJAMIN BLANDER
}

\begin{abstract}
We give a model structure on the category of simplicial presheaves on some essentially small Grothendieck site $T$. When $T$ is the Nisnevich site it specializes to a proper simplicial model category with the same weak equivalences as in $[\mathrm{MV}]$, but with fewer cofibrations and consequently more fibrations. This allows a simpler proof of the comparison theorem of [V2], one which makes no use of $\Delta$-closed classes.
\end{abstract}

The purpose of this note is to introduce different model structures on the categories of simplicial presheaves and simplicial sheaves on some essentially small Grothendieck site $T$ and to give some applications of these simplified model categories. In particular, we prove that the stable homotopy categories $\mathrm{SH}\left((\mathrm{Sm} / k)_{\mathrm{Nis}}, \mathbb{A}^{1}\right)$ and $\mathrm{SH}\left((\mathrm{Sch} / k)_{\mathrm{cdh}}, \mathbb{A}^{1}\right)$ are equivalent. This result was first proven by Voevodsky in [V2] and our proof uses many of his techniques, but it does not use his theory of $\Delta$-closed classes developed in [V3].

\section{The LOCAL PROJECTIVE MOdel STRUCTURE ON PRESHEAVES}

We first recall some of the other well-known model structures on simplicial presheaves.

Definition 1.1. A map $f: X \rightarrow Y$ of simplicial presheaves (or sheaves) is a local weak equivalence if $f_{*}: \pi_{0}(X) \rightarrow \pi_{0}(Y)$ induces an isomorphism of associated sheaves and, for all $U \in T, f_{*}: \pi_{n}(X, x) \rightarrow \pi_{n}(Y, f(x))$ induces an isomorphism of associated sheaves on $T / U$ for any choice of basepoint $x \in X(U)$. The map $f$ is a sectionwise weak equivalence (respectively sectionwise fibration) if for all $U \in T$, the map $f(U): X(U) \rightarrow Y(U)$ is a weak equivalence (respectively Kan fibration) of simplicial sets.

Heller [He] discovered a model structure on simplicial presheaves whose weak equivalences are the sectionwise weak equivalences. We will refer to his model structure as the injective model structure.

Date: January 11, 2001.

I would like to thank Dan Isaksen for his many helpful suggestions, and I thank my adviser Peter May for his encouragement and careful reading of many drafts. I am also grateful to Vladimir Voevodsky for noticing an error in an earlier version and for his work that inspired this note. 
Theorem 1.2. (Heller) The category of simplicial presheaves on a small site forms a proper simplicial cofibrantly generated model category if we define weak equivalences to be sectionwise weak equivalences, cofibrations to be monomorphisms, and fibrations to be those maps with the right lifting property with respect to acyclic cofibrations.

The first model structure on simplicial presheaves whose weak equivalences are the local weak equivalences was discovered by Jardine in [J], and in the case of sheaves by Joyal [Jo]. These are the local analogues of the injective model structure and we will refer to them as the local injective model structures.

Theorem 1.3. (Jardine) The category of simplicial presheaves on a small site forms a proper simplicial cofibrantly generated model category if we define weak equivalences to be the local weak equivalences, cofibrations to be monomorphisms, and fibrations to be those maps with the right lifting property with respect to all acyclic cofibrations.

Theorem 1.4. (Joyal) The category of simplicial sheaves on a small site forms a proper simplicial cofibrantly generated model category with weak equivalences, cofibrations and fibrations defined exactly as in the previous theorem.

The local projective model structure on presheaves is based on the following model structure, which we will refer to as the projective model structure. The following theorem is proved in [Hi], which also contains the definition of a cellular model category.

Theorem 1.5. (Hirschhorn-Bousfield-Kan-Quillen) The category of presheaves on a small site forms a proper simplicial cellular model category if we define weak equivalences to be sectionwise weak equivalences, fibrations to be sectionwise fibrations, and cofibrations to be those maps with the left lifting property with respect to acyclic fibrations.

By definition, any cellular model category is cofibrantly generated, and in this case we can explicitly describe the generating cofibrations and acyclic cofibrations. The cofibrations turn out to be exactly the retracts of transfinite compositions of pushouts along the maps

$$
\partial \Delta^{n} \otimes X \rightarrow \Delta^{n} \otimes X
$$

where $X$ is an object of $T$ considered as a represented presheaf, the simplicial sets are to be thought of as constant presheaves, and $\otimes$ is just the product (technically, $\otimes$ is part of the simplicial structure). Acyclic cofibrations are generated in the same fashion by the maps

$$
\Lambda_{k}^{n} \otimes X \rightarrow \Delta^{n} \otimes X
$$

If we "localize" this with respect to the class of local weak equivalences, we obtain the following local projective model structure for presheaves. This is 
an analog for presheaves of the model structure discovered by Brown and Gersten in [BG].

Theorem 1.6. Let $T$ be any essentially small site. Then the category $\Delta^{o p}(\operatorname{PreShv}(T))$ of presheaves of simplicial sets on $T$ admits a proper simplicial cellular model structure if we define weak equivalences to be local weak equivalences, cofibrations to be projective cofibrations, and fibrations to be those maps with the right lifting property with respect to acyclic cofibrations.

Proof. Axioms MC1 - MC3 are immediate. Let us first prove that the factorization axiom MC4 holds. Let $f: X \rightarrow Y$ be the map to be factored. Observe that any local injective fibration is a local projective fibration, since any projective cofibration is a local injective cofibration. Consequently any local projective cofibration is a local injective cofibration. Also, any acyclic projective fibration is a local weak equivalence and has the right lifting property with respect to all local projective cofibrations and therefore with respect to all acyclic local projective cofibrations, and is therefore an acyclic local projective fibration.

First factor $f$ as

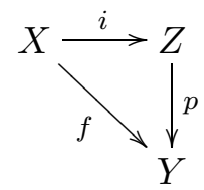

where $i$ is a projective cofibration and $p$ is an acyclic projective fibration. This provides one of the desired factorizations.

For the other factorization, use the local injective model structure to factor $f$ as

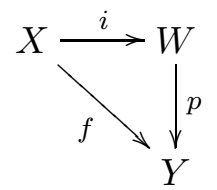

where $i$ is an acyclic local injective cofibration and $p$ is a local injective, and hence local projective, fibration. Next factor $i$ as $i=j \circ k$, where $k$ is a (necessarily acyclic) local projective cofibration and $j$ is an acyclic local projective fibration. Then $f=(p \circ j) \circ k$ yields the desired factorization.

The acyclic cofibration / fibration half of the lifting axiom MC5 is immediate from the definitions. Suppose we have a square

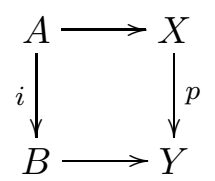

where $i$ is a cofibration and $p$ is an acyclic fibration. Factor $p$ as

$$
X \stackrel{q}{\longrightarrow} Z \stackrel{r}{\longrightarrow} Y
$$


where $q$ is a projective cofibration which is necessarily a local weak equivalence and $r$ is an acyclic projective fibration. Consider the diagram

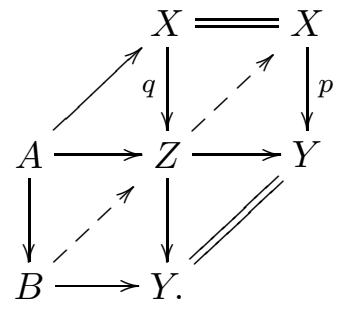

By the projective lifting axiom, a lift $B \rightarrow Z$ exists. A lift $Z \rightarrow X$ also exists since $q$ is a local projective acyclic cofibration and $p$ is a local projective fibration. The composite of these two lifts yields the desired lift in the original diagram. The following three lemmas complete the proof.

Lemma 1.7. The local projective model structure is proper.

Proof. The local projective model structure is left proper since every local projective cofibration is a local injective cofibration and the local injective model structure is left proper.

Any local projective fibration is a projective fibration, and any projective fibration is a local fibration. By [J2, Proposition 1.4] the pullback of a local weak equivalence by a local fibration (see $[\mathrm{J}]$ for a definition) is a local weak equivalence. Therefore the local projective model structure is right proper.

Lemma 1.8. The local projective model structure is simplicial.

Proof. Define a simplicial mapping object by $\operatorname{Map}(X, Y)_{n}=\operatorname{Hom}(X \times$ $\left.\Delta^{n}, Y\right)$ If $X$ is a simplicial presheaf and $K$ is a simplicial set, define $X \otimes K=$ $X \times K$, where $K$ is considered as a constant simplicial presheaf. Also define the cotensor $X^{K}$ to be $\underline{\operatorname{Hom}}(K, X)$, where $\underline{\text { Hom }}$ denotes the internal presheaf Hom. It is standard that these satisfy the usual adjunctions at the non-localized level.

To prove that the local projective model structure is simplicial, it suffices to verify axiom SM7: if $i: A \rightarrow B$ is a cofibration and $p: X \rightarrow Y$ is a fibration, then $\operatorname{Map}(B, X) \rightarrow \operatorname{Map}(A, X) \times_{\operatorname{Map}(A, Y)} \operatorname{Map}(B, Y)$ is a fibration (of simplicial sets) which is acyclic if either $i$ or $p$ is.

Since local projective cofibrations are projective cofibrations and (acyclic) local projective fibrations are (acyclic) projective fibrations, we are reduced to proving that the above map is an acyclic fibration in case $i$ is an acyclic cofibration and $p$ is a fibration.

By adjunction, it suffices to prove that for any monomorphism $K \rightarrow L$ of simplicial sets, the map

$$
B \otimes K \amalg_{A \otimes K} A \otimes L \rightarrow B \otimes L
$$

is an acyclic cofibration. It is a cofibration by the simplicial axiom SM7 for the projective model structure. It is a weak equivalence because the 
maps $A \otimes K \rightarrow B \otimes K$ and $A \otimes L \rightarrow B \otimes L$ are local projective acyclic cofibrations.

Lemma 1.9. The local projective model structure is cellular, and in particular is cofibrantly generated.

Proof. The maps $\partial \Delta^{n} \otimes U \rightarrow \Delta^{n} \otimes U$, where $U$ runs through all objects of $T$ viewed as represented presheaves, are a set of generating cofibrations. Let $A_{\alpha} \rightarrow B_{\alpha}$ be a set of generating acyclic cofibrations for the local injective model structure and choose projective cofibrant replacements $\widetilde{A_{\alpha}} \rightarrow \widetilde{B_{\alpha}}$. We will show that the local projective model structure is the left Bousfield localization of the projective model category with respect to the cofibrations $\widetilde{A_{\alpha}} \rightarrow \widetilde{B_{\alpha}}$. Therefore, by the localization machinery of [Hi, Chapter 3$]$, it will follow that the local projective model structure is cellular.

To prove the localization claim, it suffices to prove that an object $Z$ is local projective fibrant if and only if it is projective fibrant and the maps $\operatorname{Map}\left(\widetilde{B_{\alpha}}, Z\right) \rightarrow \operatorname{Map}\left(\widetilde{A_{\alpha}}, Z\right)$ are weak equivalences.

By the simplicial axiom SM7, the "only if" direction is true. Therefore, suppose $Z$ is projective fibrant and the maps above are weak equivalences.

Let $Z \rightarrow \widehat{Z}$ be an injective fibrant replacement. Since both $Z$ and $\widehat{Z}$ are projective fibrant, $\operatorname{Map}\left(\widetilde{B_{\alpha}}, Z\right) \rightarrow \operatorname{Map}\left(\widetilde{B_{\alpha}}, \widehat{Z}\right)$ is a weak equivalence and similarly for $\widetilde{A_{\alpha}}$. Therefore $\operatorname{Map}\left(\widetilde{B_{\alpha}}, \widehat{Z}\right) \rightarrow \operatorname{Map}\left(\widetilde{A_{\alpha}}, \widehat{Z}\right)$ is a weak equivalence, and by axiom SM7 for the injective model structure, we obtain that $\operatorname{Map}\left(B_{\alpha}, \widehat{Z}\right) \rightarrow \operatorname{Map}\left(A_{\alpha}, \widehat{Z}\right)$ is a weak equivalence. Therefore $\widehat{Z} \rightarrow *$ has the right lifting property with respect to $A_{\alpha} \rightarrow B_{\alpha}$ and is local injective fibrant.

Now, let $C \rightarrow D$ be any local projective acyclic cofibration. To prove that $Z$ is local projective fibrant, it suffices to show that $\operatorname{Map}(D, Z) \rightarrow \operatorname{Map}(C, Z)$ is a weak equivalence. Since local projective acyclic cofibrations are also local injective acyclic cofibrations, we $\operatorname{know} \operatorname{Map}(D, \widehat{Z}) \rightarrow \operatorname{Map}(C, \widehat{Z})$ is a weak equivalence. But we also know that $\operatorname{Map}(C, \widehat{Z}) \simeq \operatorname{Map}(C, Z)$ and similarly for $D$.

Remark 1.10. Hirschowitz and Simpson examined the local projective model structure for presheaves in the context of stacks in their preprint [HS]. Dan Dugger has also examined the local projective model structure for presheaves and his preprint $[D]$ contains statements of many of his results, which overlap with some results in this note. He has proposed a complete description of the fibrant objects in the local projective model category, and has proposed several other applications to motivic homotopy theory. For example, Dugger shows that the local projective model structure makes it possible to construct a Quillen pair

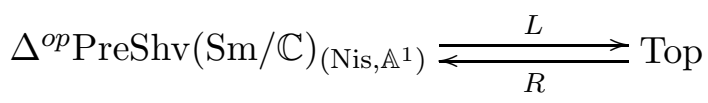


where $L$ is the geometric realization functor. Dan Isaksen and the author have an alternative construction using the characterization of fibrant objects given in Lemma 4.1 below.

\section{The local PRojective Model StRucture on SheaVeS}

The main result of this section is the following theorem.

Theorem 2.1. The category of simplicial sheaves on a small site is a proper simplicial cellular model category if we define weak equivalences to be the local weak equivalences, fibrations to be the local projective fibrations of the underlying presheaves, and cofibrations to be those maps with the left lifting property with respect to acyclic fibrations.

We also have the following straightforward but important result which, for the purposes of homotopy theory, allows us to consider categories of presheaves instead of sheaves. Let $a$ denote the associated sheaf functor.

Theorem 2.2. The functor $a$ is the left adjoint of a Quillen equivalence between the local projective model categories of presheaves and sheaves, which therefore have equivalent homotopy categories. The same is true for the local injective model categories.

Proof. This follows from the facts that the forgetful functor from sheaves to presheaves preserves fibrations and acyclic fibrations, and that for any presheaf $F$, the natural map $F \rightarrow a F$ is a local weak equivalence by [J, Lemma 2.6]. Indeed, if $F$ is a simplicial presheaf and $G$ is a simplicial sheaf, a map $a F \rightarrow G$ is a local weak equivalence if and only if $F \rightarrow G$ is.

Remark 2.3. The local projective model structure for sheaves is different than the one considered by Voevodsky in [V1]: it has more fibrations and fewer cofibrations. For example, let us consider the case of the Zariski topology on the site $\mathrm{Sm} / k$. In Voevodsky's model structure, all open inclusions of schemes, viewed as monomorphisms of represented sheaves, are cofibrations. This is not the case in the model structure of Theorem 2.1. Since a map $F \rightarrow *$ is a local projective acyclic fibration of sheaves if and only if $F(U)$ is a contractible Kan simplicial set for all $U \in T$, we need not have that $F(X) \rightarrow F(U)$ is surjective. Therefore the map $U \rightarrow X$ does not have the left lifting property with respect to the acyclic fibration $F \rightarrow *$. However, in Voevodsky's model structure, in order for $F \rightarrow *$ to be an acyclic fibration, each map $F(X) \rightarrow F(U)$ must be an acyclic fibration.

Proof of 2.1. Axioms MC1, MC2 and MC3 are obvious since fibrations and cofibrations are defined by lifting properties. We begin by proving the factorization axiom MC5, following a trick of Joyal (see $[\mathrm{J}]$ ). Let $f: X \rightarrow Y$ be a map of simplicial sheaves. First we wish to factor $f$ as a cofibration followed by an acyclic fibration. Following the trick of Joyal, construct, using transfinite induction, a filtered sequence of sheaves indexed by ordinal numbers, together with maps to $Y$ making the evident triangles commute, 
as follows. Define $X_{0}=X$, and assume the sequence is defined up to $X_{\alpha}$. Factor the map $X_{\alpha} \rightarrow Y$ as follows:

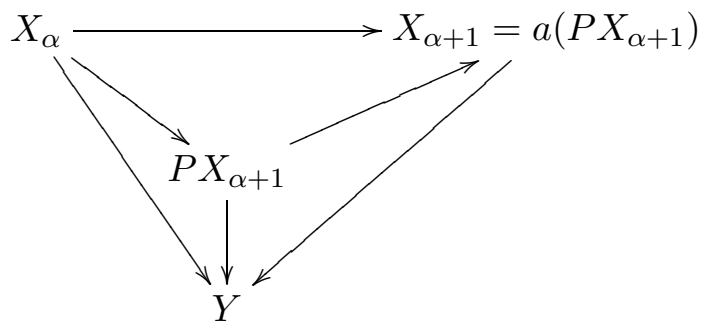

where $P X_{\alpha+1}$ is a simplicial presheaf, $X_{\alpha} \rightarrow P X_{\alpha+1}$ is a local projective cofibration, $P X_{\alpha+1} \rightarrow Y$ is an acyclic local projective fibration, and $X_{\alpha+1} \rightarrow Y$ is the map induced by the universal property of sheafification. By Theorem 2.2 , the map $X_{\alpha} \rightarrow X_{\alpha+1}$ is a cofibration. For each limit ordinal $\beta$, define $X_{\beta}=\operatorname{colim}_{\alpha<\beta} X_{\alpha}$. Since cofibrations are determined by a left lifting property, the map $X_{0} \rightarrow X_{\alpha}$ is a cofibration for any ordinal $\alpha$. We show that for $\alpha$ sufficiently large, the map $X_{\alpha} \rightarrow Y$ is an acyclic fibration. Let $\left\{A_{\gamma} \rightarrow B_{\gamma}\right\}$ be a set of small acyclic cofibrant generators for the local projective model structure on presheaves. Consider the diagram

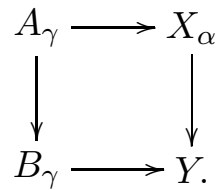

If we choose $\alpha$ to be a sufficiently large limit ordinal, the map $A_{\gamma} \rightarrow X_{\alpha}$ will factor through some $X_{\beta}$, with $\beta<\alpha$. The map $P X_{\beta+1} \rightarrow Y$ is an acyclic local projective fibration, yielding a lift $B_{\gamma} \rightarrow P X_{\beta+1}$. After composing with the map $P X_{\beta+1} \rightarrow X_{\beta+1} \rightarrow X_{\alpha}$ we obtain the desired lift in the original diagram.

Next, we must factor $f$ as an acyclic cofibration followed by a fibration. Construct sheaves $X_{\alpha}$ as before, now requiring that the map $X_{\alpha} \rightarrow X_{\alpha+1}$ be an acyclic cofibration and the map $P X_{\alpha} \rightarrow Y$ be a local projective fibration. Since the map from a presheaf to its associated sheaf is always a local weak equivalence, and since simplicial homotopy groups commute with (filtered) colimits, the map $X \rightarrow X_{\alpha}$ is an acyclic cofibration. It is also clear, by adjunction, that this map has the left lifting property with respect to all local projective fibrations of sheaves. The proof that, for sufficiently large $\alpha$, the map $X_{\alpha} \rightarrow Y$ is a fibration is exactly as before.

Finally, we must prove that the lifting axiom MC4 holds. The cofibration / acyclic fibration half is tautological, hence we must show that a lift exists in any diagram

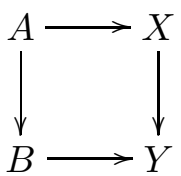


such that $A \rightarrow B$ is an acyclic cofibration and $X \rightarrow Y$ is a fibration. The proof of MC5 given above shows that we can factor the map $A \rightarrow B$ as

$$
A \stackrel{j}{\longrightarrow} Z \stackrel{i}{\longrightarrow} B
$$

where $j$ is an acyclic cofibration which has the left lifting property with respect to all fibrations, and $i$ is a (necessarily acyclic) fibration. Therefore, by the tautological half of MC4, a lift exists in the diagram

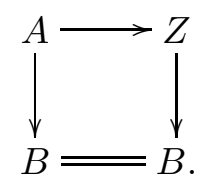

This gives the retraction

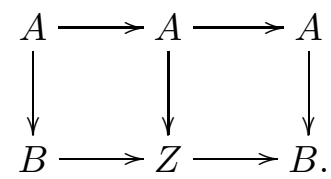

The map $A \rightarrow B$ is a retract of a map which has the left lifting property with respect to all fibrations, hence $A \rightarrow B$ also has the left lifting property with respect to all fibrations, proving MC4.

To prove that this model structure is left proper, notice that any cofibration of sheaves is also a local injective cofibration of sheaves (i.e. a sectionwise monomorphism). Thus since the local injective model structure is left proper, the local projective model structure on sheaves is also.

Right properness follows immediately from the corresponding result for presheaves.

To see that the model structure is simplicial, use [GJ, Chapter 2, Proposition 3.13] and the fact that fibrations of sheaves are exactly local projective fibrations of the underlying presheaves.

In the proof we showed that the sheafifications of generating local projective (acyclic) cofibrations of presheaves are generating (acyclic) cofibrations of sheaves. This proves that the model structure is cellular.

\section{Sites With INTERVAL}

In this section, we assume familiarity with the definition of a site with interval [MV, Section 2.3]. We also assume that all represented presheaves are sheaves, but this assumption is only necessary when we are concerned with model structures for simplicial sheaves. For simplicity, we also assume that the interval sheaf $I$ and the terminal sheaf $*$ are represented by objects of $T$. These conditions hold in most cases of interest. We begin with the following definition.

Definition 3.1. A simplicial presheaf $Z$ is $I$-local if it is local projective fibrant and for all objects $U \in T$, the maps $\operatorname{Map}(U \times I, Z) \rightarrow \operatorname{Map}(U, Z)$ are weak equivalences. A map $f: X \rightarrow Y$ of simplicial presheaves is an 
$I$-local weak equivalence if for every $I$-local $Z$, the map $f^{*}: \operatorname{Map}(Y, Z) \rightarrow$ $\operatorname{Map}(X, Z)$ is a weak equivalence.

Theorem 3.2. The categories of presheaves and sheaves on a site $T$ with interval I admit proper simplicial cellular model structures if we define cofibrations to be local projective cofibrations of (pre)sheaves, weak equivalences to be I-local weak equivalences, and fibrations to be those maps with the right lifting property with respect to acyclic cofibrations.

We also have the following analogue of Theorem 2.2.

Theorem 3.3. The functor $a$ is the left adjoint of a Quillen equivalence between the I-local projective categories of presheaves and sheaves, which therefore have equivalent homotopy categories.

Using the localization machinery of [Hi, Chapter 3], we can form the left Bousfield localization of the local projective model structure on (pre)sheaves on the site $T$ at the maps $U \rightarrow U \times I$ defined by base change along the given map $* \rightarrow I$. Note that all objects in sight are cofibrant since they are represented presheaves. This yields the model structure of Theorem 3.2, and results in [Hi, Chapter 3] show that it is left proper, simplicial and cellular. From here on, we will refer to this model structure as the $I$-local projective model structure. We now prove that it is right proper.

Lemma 3.4. The I-local projective model structure is right proper.

Proof. Suppose we have a pullback square

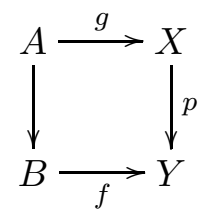

such that $p$ is an $I$-local projective fibration and $f$ is an $I$-local weak equivalence. We wish to show that $g$ is an $I$-local weak equivalence. Since the local injective model structure is right proper, by [Hi, Proposition 11.2.10] there exists a pullback diagram

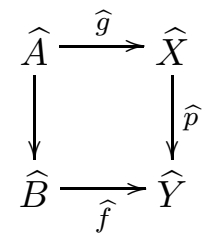

that is a local injective fibrant approximation to the original diagram. We may further assume that $\widehat{p}$ is a local injective fibration. We have reduced to showing that the map $\widehat{g}$ is an $I$-local weak equivalence. By the right properness of the $I$-local injective model structure [MV, Theorem 2.3.2], it suffices to show that the map $\widehat{p}$ is an $I$-local injective fibration. 
The reader is warned that the following arguments are technical and are not relevant to the rest of the note; they may be skipped on a first reading. Let $A_{i} \rightarrow B_{i}$ be a set of generating acyclic $I$-local injective cofibrations and let $\widetilde{A_{i}} \rightarrow \widetilde{B_{i}}$ be local projective cofibrations which are their local projective cofibrant replacements. The map $p$ has the right lifting property with respect to the maps $\widetilde{A_{i}} \rightarrow \widetilde{B_{i}}$. It suffices to show that the map $\widehat{p}$ also has the right lifting property with respect to those maps. Indeed, if this holds, the left properness of the local injective model structure and [Hi, Proposition 11.1.18] imply that $\widehat{p}$ has the right lifting property with respect to the maps $A_{i} \rightarrow B_{i}$ and is therefore an $I$-local injective fibration.

For simplicity of notation, choose a representative $A \rightarrow B$ from the set of maps $\widetilde{A_{i}} \rightarrow \widetilde{B_{i}}$. Also let the symbol " $\simeq$ " denote a local weak equivalence and let the symbol " $\simeq$ " denote an $I$-local weak equivalence. Denote local projective cofibrations by the arrow " $\longrightarrow$ " and denote local projective fibrations by the arrow " $\longrightarrow$." Finally, denote $I$-local projective fibrations by the arrow " $\stackrel{I-\text { local }}{\longrightarrow}$. By the pushout diagram

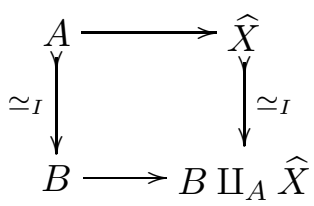

we may assume without loss of generality that $A=\widehat{X}$ and we are reduced to finding a lift in a diagram of the form

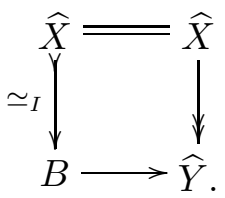

If we factor the map $B \rightarrow \widehat{Y}$ as a local projective acyclic cofibration followed by a local projective fibration, we may further assume that the map $B \rightarrow \widehat{Y}$ is a local projective fibration. Consider the pullback $B \times_{\widehat{Y}} Y$. By right properness of the local projective model structure, the map $B \times_{\widehat{Y}} Y \rightarrow B$ is a local weak equivalence. Therefore the map $X \rightarrow B \times_{\widehat{Y}} Y$ induced by the universal property is an $I$-local weak equivalence. Factor that map as a (necessarily $I$-local acyclic) projective cofibration followed by a local projective acyclic fibration:

$$
X \stackrel{\simeq_{I}}{\longrightarrow} C \stackrel{\simeq}{\longrightarrow} B \times_{\widehat{Y}} Y
$$


We then have the diagram

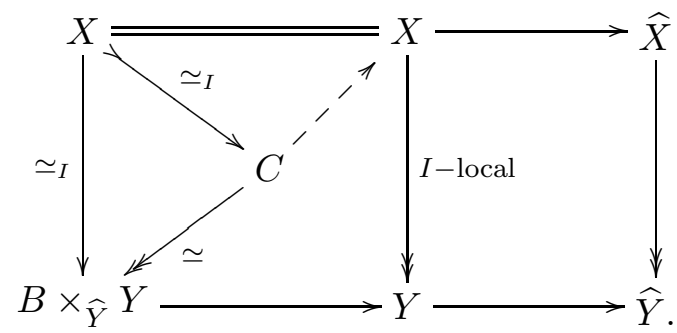

Therefore the map $\widehat{X} \rightarrow \widehat{Y}$ has the right lifting property with respect to the map $X \rightarrow C$. By virtue of the left properness of the local projective model structure, [Hi, Proposition 11.1.18], and the square

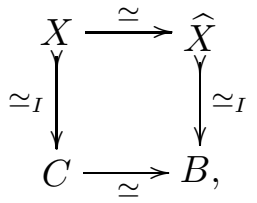

the map $\widehat{X} \rightarrow \widehat{Y}$ has the right lifting property with respect to the map $\widehat{X} \rightarrow B$.

The following lemma provides a description of the fibrant objects in this model structure.

Lemma 3.5. An object $F$ is fibrant for the I-local projective model structure if and only if $F$ is local projective fibrant and $F(U \times I) \rightarrow F(U)$ is a weak equivalence for all $U \in T$.

Proof. This follows from Hirschhorn's construction of left Bousfield localization: an object $F$ is fibrant for the $I$-local projective model structure if and only if it is fibrant for the local projective model structure and for all $U \in T$, the maps $\operatorname{Map}(U \times I, F) \rightarrow \operatorname{Map}(U, F)$ are weak equivalences, which implies the conclusion.

The following lemma characterizes weak equivalences between fibrant objects in the $I$-local projective model structure.

Lemma 3.6. Let $F$ and $G$ be fibrant simplicial presheaves in the I-local projective model structure. Then a map $F \rightarrow G$ is an I-local weak equivalence if and only if it is a projective weak equivalence.

Proof. Any projective weak equivalence is clearly an $I$-local weak equivalence. Suppose $F \rightarrow G$ is an $I$-local weak equivalence. Then since $F$ and $G$ are fibrant for the $I$-local projective model structure, for any $U \in T$ the map $\operatorname{Map}(U, F) \rightarrow \operatorname{Map}(U, G)$ is a weak equivalence. Therefore $F \rightarrow G$ is a projective weak equivalence. 


\section{Specialization to CD-TOpologies}

In this section, we consider the special case when the topology on the site in question is defined by a cd-structure in the sense of [V1]. This allows us to use the techniques developed in [V1] to obtain a very concrete description of the fibrations in the local projective model structure for presheaves.

Lemma 4.1. Let $T$ be a site with an initial object whose topology is defined by a complete bounded regular cd-structure. Then a simplicial presheaf $F$ is local projective fibrant if and only if $F(U)$ is a Kan simplicial set for all $U \in T$ and for every distinguished square

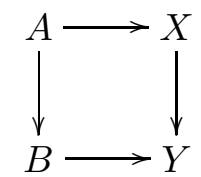

the square

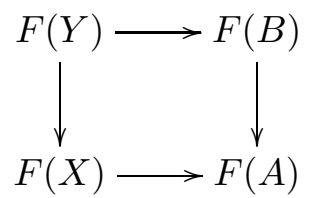

is a homotopy pullback of simplicial sets. (Such presheaves are called flasque).

Proof. In any topology defined by a cd-structure, the empty sieve is a covering sieve of the initial object $\emptyset$. We require that the "degenerate square" with only one entry, $\emptyset$, in the lower right hand corner, be a distinguished square. Therefore, if $F$ is flasque, $F(\emptyset)$ is weakly equivalent to the homotopy pullback of the empty diagram and is contractible.

Let $F$ be a local projective fibrant simplicial presheaf. Clearly $F$ is projective fibrant. Let $G$ be a fibrant replacement of $a F$ in the local injective model structure on sheaves. Then the composite map $F \rightarrow G$ is a local weak equivalence, and since the local injective model structure for presheaves has more cofibrations than the local projective model structure, $G$ is local projective fibrant when considered as a presheaf. Let $U$ be any represented presheaf. Since $F$ and $G$ are fibrant and $U$ is cofibrant, $S(U, F) \rightarrow S(U, G)$ is a weak equivalence. Therefore $F(U) \rightarrow G(U)$ is a weak equivalence for any $U$. Since the cd-structure is regular, [V1, Corollary 2.16] implies that any distinguished square is a homotopy pushout in the local injective model structure. Therefore, by adjunction, $G$ is flasque. Since the property of being flasque is invariant under projective weak equivalences of projective fibrant presheaves, $F$ is also flasque.

Conversely, suppose $F$ is a flasque simplicial presheaf. Construct the map $F \rightarrow G$ as above. Since $F$ and $G$ are both flasque, $F \rightarrow G$ is a projective weak equivalence by [V1, Lemma 3.5]. By the discussion above, $G$ is a local projective fibrant presheaf. Let $A \rightarrow B$ be an acyclic local projective cofibration of presheaves. Then $\operatorname{Map}(B, F) \simeq \operatorname{Map}(B, G) \simeq \operatorname{Map}(A, G) \simeq$ 
$\operatorname{Map}(A, F)$ since $F$ and $G$ are projective fibrant and since $G$ is local projective fibrant. Therefore we conclude that $F$ is local projective fibrant.

Remark 4.2. This differs from the description of fibrant objects proposed in $[D]$, which uses hypercoverings instead of just coverings. We expect that the two descriptions are the same in the case of a topology defined by a complete bounded regular cd-structure, but the (more complicated) description in [D] is expected to work for arbitrary sites.

We now wish to describe the cofibrant generators of the local projective model structure in case the topology is defined by a complete bounded regular cd-structure. The generating cofibrations are all maps of the form

$$
\partial \Delta^{n} \otimes X \rightarrow \Delta^{n} \otimes X
$$

for $X \in T$. Let $\alpha$ denote a distinguished square

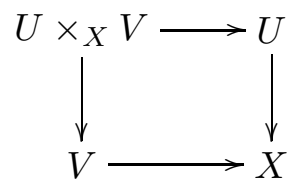

and denote the homotopy pushout of the diagram

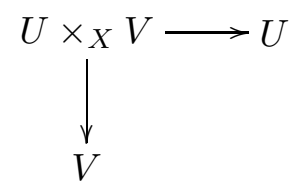

in the projective model structure by $P(\alpha)$. There is a natural map $P(\alpha) \rightarrow$ $X$, and both objects are cofibrant. In the case of the "degenerate square" described in the proof of Lemma 4.1, this map is the map from the empty sheaf to the sheaf represented by the initial object $\emptyset$ of the site $T$. We have the following lemma.

Lemma 4.3. The local projective model category is the left Bousfield localization of the projective model category with respect to the maps $P(\alpha) \rightarrow X$.

Proof. By the definition of left Bousfield localization, it suffices to show that the fibrant objects we obtain by localization are the same as those of Lemma 4.1. An object $F$ is local projective fibrant if and only if it is projective fibrant and the maps $\operatorname{Map}(X, F) \rightarrow \operatorname{Map}(P(\alpha), F)$ are weak equivalences. Indeed, by adjunction, these maps are weak equivalences if and only if the squares displayed in Lemma 4.1 are homotopy pullbacks. Lemma 4.1 completes the proof.

The following corollary, which completely describes the local projective fibrations, follows from Lemma 4.3 and the definition of left Bousfield localization. 
Corollary 4.4. A map $F \rightarrow G$ is a local projective fibration if and only if it is a projective fibration and the natural maps

$$
F(X) \rightarrow(\operatorname{holim} F(\alpha)) \times_{\operatorname{holim} G(\alpha)} G(X)
$$

are weak equivalences.

Using the description of generating acyclic cofibrations in left Bousfield localizations of [Hi, Chapter 3], we obtain the following concrete description of the generating acyclic cofibrations for the local projective model structure. Let $A_{\alpha} \rightarrow B_{\alpha}$ be cofibrant replacements of the morphisms $P(\alpha) \rightarrow X$. Then generating acyclic cofibrations for the local projective model structure have the form

$$
\Lambda_{k}^{n} \otimes X \rightarrow \Delta^{n} \otimes X
$$

and

$$
\left(A_{\alpha} \otimes \Delta^{n}\right) \amalg_{A_{\alpha} \otimes \partial \Delta^{n}}\left(B_{\alpha} \otimes \partial \Delta^{n}\right) \rightarrow B_{\alpha} \otimes \Delta^{n} .
$$

\section{COMPARISON}

The results above on local projective model structures have obvious pointed analogues, which we can use to construct stable homotopy categories in the usual way. The goal of this section is to prove the following theorem.

Theorem 5.1. The s-stable motivic homotopy categories in the scdh and Nisnevich topologies have the same fibrant objects and the same cofibrations, and are therefore equivalent. The same is true for the T-stable motivic homotopy categories.

By [V2, Lemma 4.6], this has the following immediate consequence.

Corollary 5.2. If $k$ admits resolution of singularities, the stable homotopy categories

$$
\mathrm{SH}\left((\mathrm{Sm} / k)_{\mathrm{Nis}}, \mathbb{A}^{1}\right)
$$

and

$$
\mathrm{SH}\left((\operatorname{Sch} / k)_{\operatorname{cdh}}, \mathbb{A}^{1}\right)
$$

are equivalent.

Using presheaves instead of sheaves substantially simplifies the proof of Theorem 5.1. Consider the commutative diagram

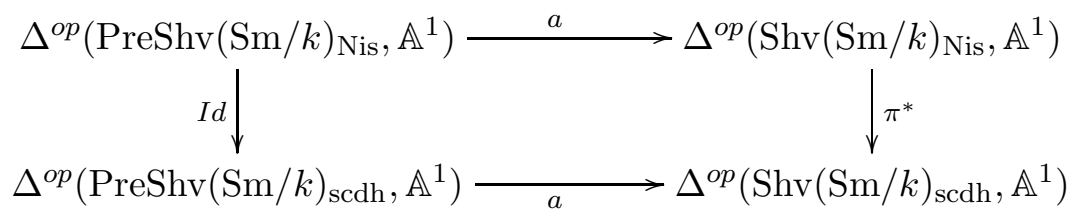

in which the horizontal arrows induce equivalences of $\mathbb{A}^{1}$-local projective homotopy categories. By virtue of this diagram, if we wish to compare 
the stable categories in the scdh and Nisnevich topologies, we only need to examine the identity functor

$$
\left(\operatorname{PreShv}(\operatorname{Sm} / k)_{\text {Nis }}, \mathbb{A}^{1}\right) \rightarrow\left(\operatorname{PreShv}(\operatorname{Sm} / k)_{\mathrm{scdh}}, \mathbb{A}^{1}\right) .
$$

This illustrates a major advantage gained by working with presheaves instead of sheaves: if we were to work with sheaves we would be forced to consider the more complicated functor $\pi^{*}$. The identity functor sends $\mathbb{A}^{1}$ Nisnevich local weak equivalences to $\mathbb{A}^{1}$-scdh local weak equivalences since the scdh topology is finer than the Nisnevich topology. The following key lemma provides a near converse.

Lemma 5.3. Let $F$ be an $\mathbb{A}^{1}$-Nisnevich local projective fibrant simplicial presheaf such that $F$ is $\mathbb{A}^{1}$-Nisnevich weakly equivalent to the simplicial loop space of an $\mathbb{A}^{1}$-Nisnevich local projective fibrant simplicial presheaf. Then $F$ is $\mathbb{A}^{1}$-scdh local projective fibrant.

Proof. Let $\mathrm{Map}_{*}$ denote the based simplicial mapping space and let $F \simeq$ $\Omega_{s} G$. By the definition of the scdh topology, it suffices to show that for any scdh distinguished square

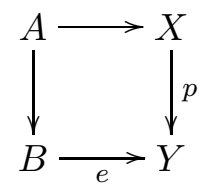

such that $e$ is a closed embedding and $p$ is the blow-up with center in $e(B)$, the square

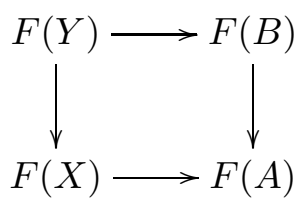

is a homotopy pullback. By [MV, Remark 3.2.30], the first simplicial suspension of the square

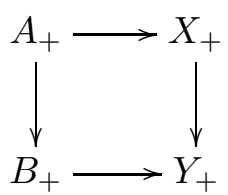

is a homotopy pushout in the $\mathbb{A}^{1}$-Nisnevich homotopy category. Here the subscript "+" means we adjoin a disjoint basepoint. Hence, by adjunction, $F(Y) \simeq \operatorname{Map}_{*}\left(\Sigma_{s}^{1}\left(Y_{+}\right), G\right)$ is weakly equivalent to the homotopy limit of the diagram

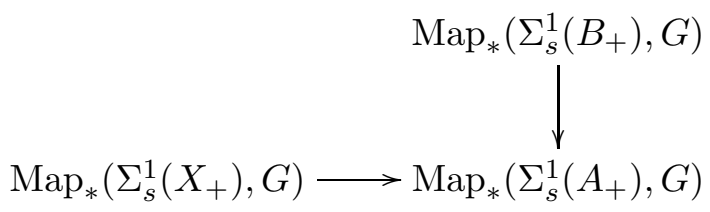


which is weakly equivalent to the homotopy limit of the diagram

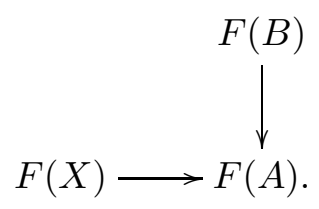

Therefore $F$ is $\mathbb{A}^{1}$-scdh local projective fibrant.

Corollary 5.4. Let $f: F \rightarrow G$ be an $\mathbb{A}^{1}$-scdh local pointed weak equivalence. Then $\Sigma_{s}^{1}(f)$ is an $\mathbb{A}^{1}$-Nisnevich local pointed weak equivalence.

Proof. Let $X$ be an $\mathbb{A}^{1}$-Nisnevich local projective fibrant pointed presheaf. Then $\Omega_{s}(X)$ is also $\mathbb{A}^{1}$-Nisnevich local projective fibrant, and by Lemma 5.3 it is $\mathbb{A}^{1}$-scdh local projective fibrant. It suffices to show that $\Sigma_{s}^{1}(f)^{*}$ : $\operatorname{Map}_{*}\left(\Sigma_{s}^{1}(G), X\right) \rightarrow \operatorname{Map}_{*}\left(\Sigma_{s}^{1}(F), X\right)$ is a weak equivalence. By adjunction, this is equivalent to showing that $\operatorname{Map}_{*}\left(G, \Omega_{s}(X)\right) \rightarrow \operatorname{Map}_{*}\left(F, \Omega_{s}(X)\right)$ is a weak equivalence, which is true since $f$ is an $\mathbb{A}^{1}$-scdh local pointed weak equivalence and $\Omega_{s}(X)$ is $\mathbb{A}^{1}$-scdh local projective fibrant.

This corollary immediately proves Theorem 5.1.

\section{REFERENCES}

[BG] K. S. Brown and S. M. Gersten, Algebraic K-theory and generalized sheaf cohomology, in Lecture Notes in Mathematics 341, 1973, pp. 266-292.

[D] D. Dugger, Universal Homotopy Theories, preprint, http://hopf.math.purdue.edu/pub/hopf.html, 2000.

[GJ] P. Goerss and J. Jardine, Simplicial Homotopy Theory, Birkhauser, 1999.

[SGA] A. Grothendieck, M. Artin, and J.-L. Verdier, Théorie des topos et cohomologie étale des schémas (SGA 4), Springer Lecture Notes in Mathematics 269, 270, 305, 1972-1973.

[He] A. Heller, Homotopy theories, Memoirs Amer. Math. Soc. Vol. 71, no. 383, 1988.

[Hi] P. Hirschhorn, Localizations of Model Categories, preprint, http://www-math.mit.edu/ ${ }^{\sim}$ psh/, 2000.

[HS] A. Hirschowitz and C. Simpson, Descente pour les n-champs, preprint, http://front.math.ucdavis.edu/, 1998.

[J] J. Jardine, Simplicial Presheaves, J. Pure and Applied Algebra 47, 1987, no. 1, 35-87.

[J2] J. Jardine, Stable homotopy theory of simplicial presheaves, Canadian J. Math. 39(3), 1987, 733-747.

[Jo] A. Joyal, Letter to A. Grothendieck, 1985.

[MV] F. Morel and V. Voevodsky, $\mathbb{A}^{1}$-Homotopy Theory of Schemes, preprint, http://www.math.uiuc.edu/K-theory, 1998.

[V1] V. Voevodsky, Homotopy theory of simplicial sheaves in completely decomposable topologies, preprint, http://www.math.uiuc.edu/K-theory, 2000.

[V2] V. Voevodsky, Unstable motivic homotopy categories in Nisnevich and cdh-topologies, preprint, http://www.math.uiuc.edu/K-theory, 2000.

[V3] V. Voevodsky, $\Delta$-closed classes, preprint, http://www.math.uiuc.edu/K-theory, 2000. 